\title{
Bereich Dienste
}

\author{
Tätigkeitsbericht 2002
}

Erika Flückiger

Der Bereich Dienste im Generalsekretariat der FMH umfasst einerseits Abteilungen, die täglich in direktem Kontakt zu den Mitgliedern oder den Basisorganisationen und anderen ärztlichen Organisationen der FMH stehen: Dienstleistungen an Ärztegesellschaften/Mitgliedschaft (DLM), Rechtsdienst, Prävention, Medizinische Praxisassistentinnen (MPA), andererseits Dienste, die durch Sicherstellung entsprechender Infrastrukturen die Abteilungen und Stabsdienste im Generalsekretariat in der Bewältigung ihrer Aufgaben unterstützen: Übersetzung, IT Support, Telefonzentrale/Empfang und Liegenschaft/Hausdienst (Abb. 2).

Die Aufgabenausrichtung dieser beiden Gruppen gibt vor, dass die zu erbringenden Dienstleistungen eine hohe Qualität aufweisen und kundenorientiert sein müssen. Oberstes Ziel ist die Zufriedenheit unserer «Kunden»: der Mitglieder der FMH und der in der FMH organisierten Gesellschaften. Kundenzufriedenheit ihrerseits geht einher mit Mitarbeiterzufriedenheit. Der Weg hin zu diesem Ziel führt über Analyse, Hinterfragung und kontinuierliche Verbesserung der Abläufe (Prozesse) sowie über die Einführung von qualitätsfördernden Massnahmen, damit in den Geschäftsprozessen Effektivität und Effizienz erreicht werden. Der weitaus grösste Teil der Aufgaben, die vom Generalsekretariat bearbeitet werden, sind abteilungsübergreifend. Nehmen wir als Beispiel dafür den vom Bundesrat im vergangenen Jahr erlassenen Zulassungsstopp: Er hat von der Telefonzentrale über das Sekretariat Aus-, Weiter- und Fortbildung, den Rechtsdienst, bis hin zum Mitgliedschaftswesen in fast allen Abteilungen mehr oder weniger tiefe Spuren hinterlassen. Eine Loslösung vom traditionellen Stellen- und Abteilungsdenken hin zur Prozessorientierung drängt sich damit auf: das «eigene Gärtchen pflegen» muss definitiv der Vergangenheit angehören. Ein wichtiges Mittel zur Analyse und Gestaltung von betrieblichen Prozessen ist die Nutzung softwaretechnischer Unterstützung. So kann etwa durch eine Automatisierung von Vorgängen mit Hilfe neuer Technologien und Computersystemen die Effizienz eines Prozesses wesentlich gesteigert werden. Ein weiteres wichtiges Mittel zur Effizienzsteigerung ist das Zurverfügungstellen eigenen
Abbildung 1

Erika Flückiger, Leiterin Dienste.

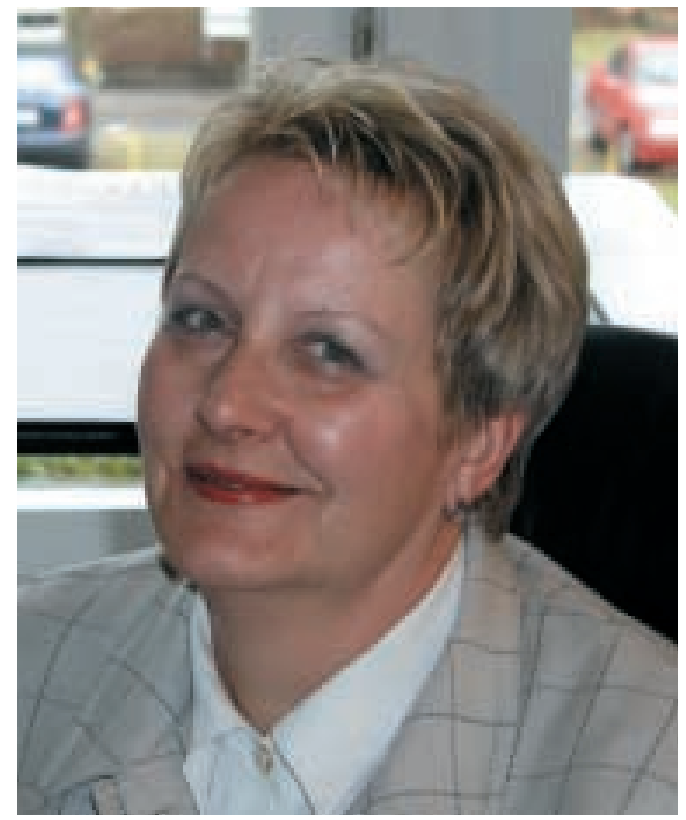

Wissens, eigener Kenntnisse und Informationen an all jene, die im Rahmen ihrer Aufgaben davon profitieren können oder darauf angewiesen sind. Vor ein paar Jahren haben wir zu diesem Zweck ein sog. Knowledge-Management-System (Livelink) beschafft und eingeführt, das uns gleichzeitig zur elektronischen Archivierung unserer geschäftsrelevanten Prozesse dient. Mit der Vergabe von Berechtigungen kann sehr einfach geregelt werden, wem anders Zugriff auf die eigenen Dateien gewährt werden soll. Auch die Informationssuche ist sehr viel einfacher und effizienter als dies im traditionellen Papierarchiv möglich wäre. Allerdings verlangt dieses neue Arbeitsinstrument von den Mitarbeitern grosse Disziplin, denn jeder einzelne ist nun selbst dafür verantwortlich, dass die eigenen Daten in diesem elektronischen Archiv gespeichert werden und nicht einfach auf der eigenen Festplatte verbleiben. Auch die Zugriffsregelung wird tendenziell noch zu restriktiv gehandhabt. Zur gegenseitigen Information und Absprache sowie zum raschen Reagieren und Ergreifen von notwendigen Massnahmen dient zudem der regelmässig wöchentlich stattfindende Rapport des 


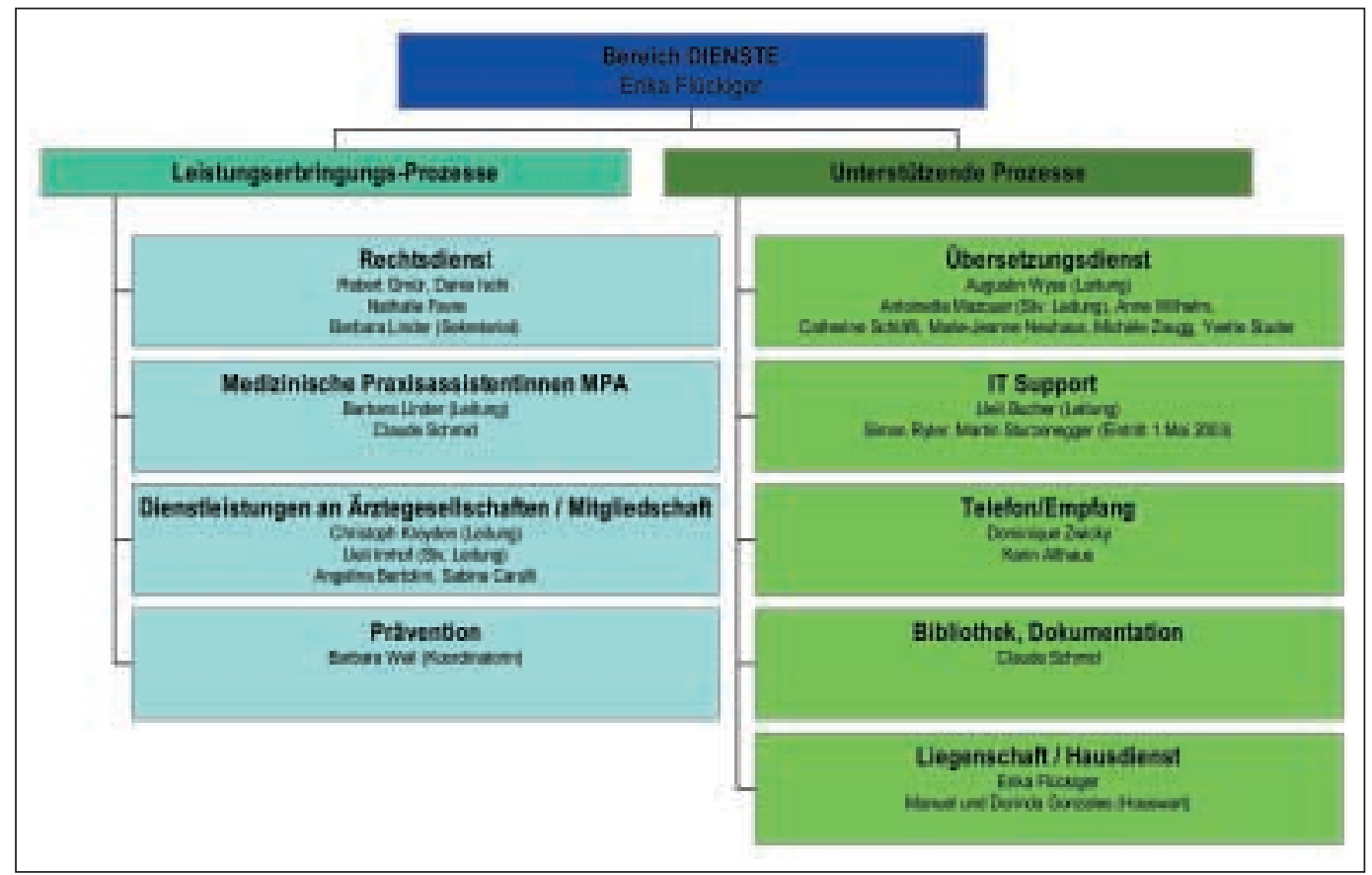

Bereichs Dienste, an dem die Verantwortlichen und zum Teil Sachbearbeiter/innen der einzelnen Abteilungen teilnehmen.

\section{FMH goes public}

Was nützt eine Dienstleistung, und sei sie noch so gut, wenn die «Kunden» sie nicht kennen?

Es ist für einen Verband, der die Interessen seiner Mitglieder vertritt, wahrt und verteidigt, nicht immer ganz einfach aufzuzeigen, welchen persönlichen und ganz konkreten Nutzen das einzelne Mitglied aus bestimmten Massnahmen ziehen kann; schwierig wird es insbesondere dann, wenn die Interessen unter den Verbandsmitgliedern stark divergieren. In dieser Beziehung haben es die Abteilungen im Bereich Dienste etwas einfacher: Wer zum Beispiel beim Rechtsdienst um eine Auskunft nachsucht, kann einen ganz konkreten Nutzen für seine spezifische Situation aus der ihm erteilten Antwort ziehen und sieht damit gleichzeitig auch einen konkreten Sinn in seiner FMH-Mitgliedschaft. Wie dieses Beispiel, gibt es zahlreiche Dienstleistungen, von denen das einzelne FMH-Mitglied kraft seiner Zugehörigkeit zum Verband profitieren kann.

Möglichkeiten, diese Dienstleistungen unter den Mitgliedern auch bekannt zu machen, gibt es beispielsweise über die Website der FMH: www.fmh.ch, oder über Artikel, Hinweise und Präsentationen in der Schweizerischen Ärztezeitung.

Eine weitere Gelegenheit, unser Angebot an vielfältigen Dienstleistungen zu unseren bestehenden und künftigen Mitgliedern zu bringen, hat sich uns im Frühling 2001 geboten: Ausgehend von einer gemeinsamen Initiative des Verbandes Schweizerischer Assistenz- und Oberärztinnen und -ärzte VSAO und des Verbandes Schweizer Medizinstudierender VSM fand im März 2001 in Bern der erste Medico-Vadis-Kongress statt. Er stand unter dem Motto: «Welche Zukunftsaussichten haben junge Medizinerinnen und Mediziner?» Selbstverständlich, dass auch die FMH sich aktiv an diesem Kongress beteiligte, einerseits mit Referaten und Workshops, andererseits auch mit einem eigenen Ausstellungsstand. Weil wir bezüglich Kongresstätigkeit Neuland betraten, entschieden wir uns für einen sehr flexiblen Stand, der aber gleichzeitig die Möglichkeit bot, später ausgebaut oder als Modul in einem grösseren Kontext verwendet und jederzeit auf einfache Art in seiner textlichen Aussage aktualisiert und an das jeweilige Zielpublikum angepasst zu werden. Auf diesen Anlass hin haben wir auch eine handliche Broschüre geschaffen, die einen kurzen Abriss über die Dienstleistungen der FMH an ihre Mitglieder enthält und auf die entsprechenden Anlaufstellen im Generalsekretariat hinweist. Das Inter- 
Abbildung 3

Ausstellungsstand am Medico-Vadis-Kongress.

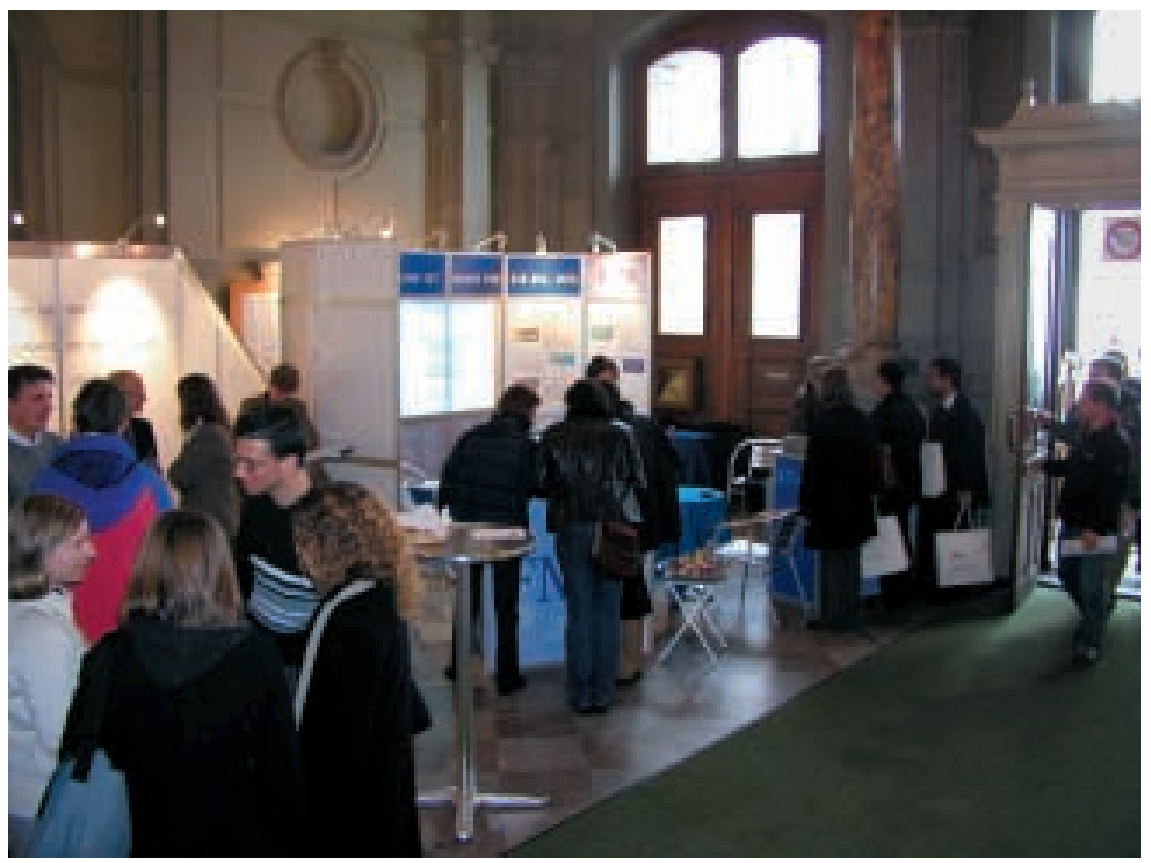

Abbildung 4

Ausstellungsstand an der IFAS.

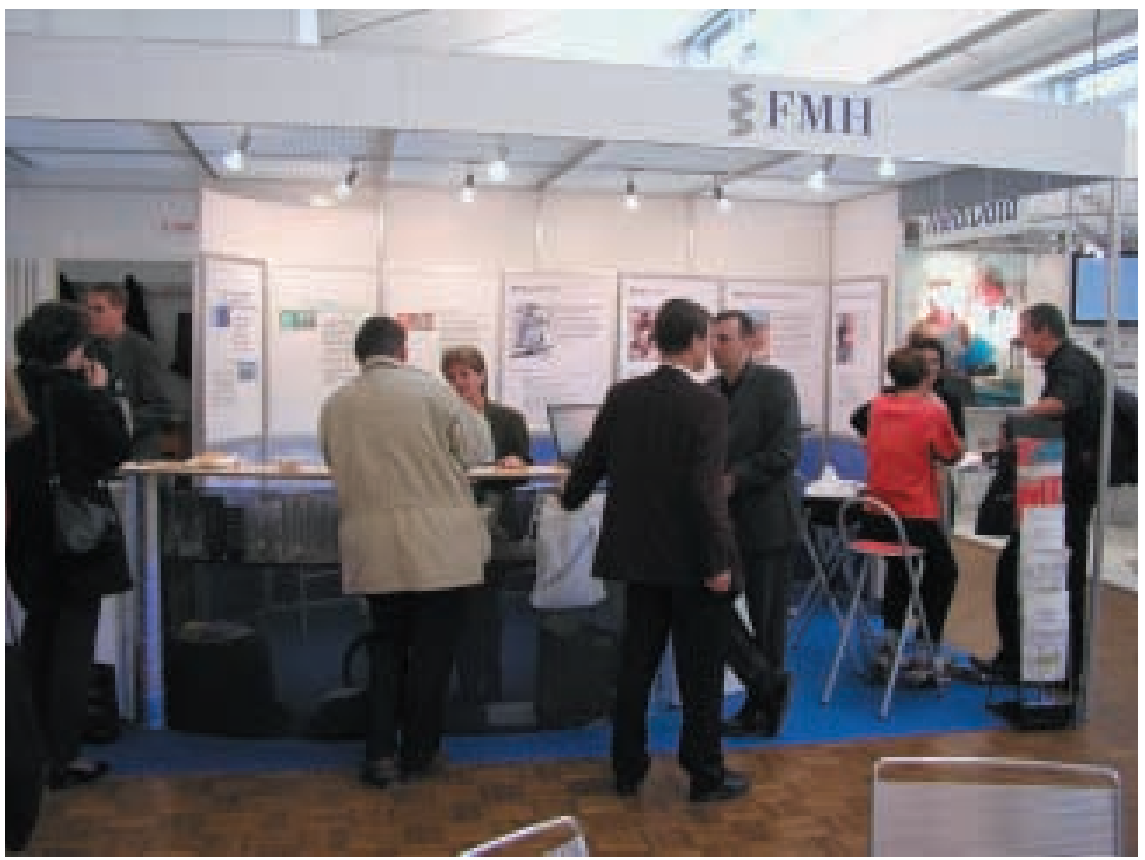

esse, auf welches wir bei den jungen Kongressteilnehmerinnen und -teilnehmern stiessen, hat uns gezeigt, dass der Entscheid sowohl bezüglich Präsenz als auch, was die Art des Ausstellungsstandes betraf, richtig war (Abb. 3). Letzteres vor allem deshalb, weil wir den Stand problemlos zu zweit und in relativ kurzer Zeit aufstellen und abbauen können, was uns in der Zwischenzeit schon etliche neidvolle Blicke von anderen Ausstellern eingetragen hat.

Seit unserer Ausstellungspremière haben wir unsere Kongresstätigkeit etwas ausgebaut: Im Jahr 2001 waren wir mit unserem Stand am Jahreskongress der Schweizerischen Gesellschaft für Allgemeinmedizin SGAM in Zürich und an dem von den FMH Services initiierten und organisierten 1. Internationalen Managed-HealthCare-Kongress in Winterthur dabei. Im Geschäftsjahr 2002 haben wir an der Jahresversammlungen der SGIM (Schweiz. Gesellschaft für Innere Medizin) in Genf, an der Jahresversammlung SGAM in Lugano und am 2. MedicoVadis-Kongress in Bern teilgenommen. Eine besondere Herausforderung für unsere Kongresstätigkeit stellte unsere Präsenz an der IFAS 2002 (Internationale Fachausstellung für Arzt- und Spitalbedarf) in Zürich dar. Für diesen Anlass, den wir - wie alle übrigen Ausstellungen - gemeinsam mit den FMH Services bestritten, nahmen wir die Unterstützung eines professionellen Standbauers in Anspruch (Abb. 4). Auf sehr gutes Echo sind die Kurzpräsentationen bzw. Workshops gestossen, die wir an allen 4 Ausstellungstagen zu folgenden Themen durchführten:

- Steueroptimierung mit Versicherungsprodukten;

- Vorzeitige Pensionierung/Finanzplanung;

- Die Arztpraxis aus steuerlicher Sicht;

- Ausstehende Honorarforderungen. Was nun?

- Gesicherte E-Mail-Kommunikation zwischen Spital und Praxis;

- «Werbung» und Websites für Arztpraxen;

- Praxisübernahme: Worauf Sie achten müssen;

- Die ärztliche Gruppenpraxis: Tips zur Vertragsgestaltung.

Im Anschluss an die Workshops wurden die Teilnehmer an unserem Stand mit einem kleinen Geschenk und einer Erfrischung überrascht. Hier bot sich dann auch die Gelegenheit, den anwesenden Mitarbeiterinnen und Mitarbeitern vertieftere Fragen zum präsentierten Workshopthema oder zu persönlichen Anliegen zu stellen und detailliertere Dokumentation mitzunehmen. Es versteht sich von selbst, dass die Workshops für FMH-Mitglieder kostenlos angeboten wurden.

Die nächste IFAS findet im Jahr 2004 statt: Die FMH und die FMH Services werden selbstverständlich wieder für die FMH-Mitglieder und solche, die es werden wollen, da sein und Interessierten auch wiederum Workshops zu aktuellen Themen anbieten. 